After $(n-1)$ modes have been found, and the reduction of the matrix $a_{r}$, carried out for each of them in the manner described, we must be left with the matrix

$$
a_{r s}-\sum_{i \neq i} a_{r s}^{(i)}=a_{r s}^{(i)},
$$

where $i$ is the number of the mode left to be found. In this matrix the terms of any row are proportional to $\sum_{s} a_{s e} x_{e}^{(i)}$ so the remaining $i$-th mode can be found by solving the set of linear equations

$$
\sum_{s} a_{r s} x_{s}=a_{1 r}^{(i)}
$$

The reductions can often be carried out with advantage in terms of the matrix $b_{r}$; this is done by normalizing the modes so that instead of satisfying (10) they satisfy

$$
\sum_{r s} b_{r s} y_{r}^{(i)} y_{s}^{(i)}=1 \text {, }
$$

and calculating $a_{r s}^{(i)}$ from the formula

$$
a_{r s}^{(i)}=\lambda^{(i)} \cdot \sum_{k} b_{r k} y_{k}^{(i)} \sum_{e} b_{s e} y_{e}^{(i)}
$$

which is easily deduced from (8). This is particularly convenient in the most usual type of problem in which $b_{r s}$ is a diagonal matrix - in mechanical problems, those in which the kinetic energy can be expressed as a sum of squares. If $\left|b_{r s}\right|=\left|m_{r} \delta_{r s}\right|$ the modes must be normalized by

$$
\sum_{r} m_{r}\left(y_{r}^{(i)}\right)^{2}=1
$$

and then

$$
a_{r s}^{(i)}=\lambda^{(i)} m_{r} m_{s} x_{r}^{(i)} x_{s}^{(i)} .
$$

After reductions corresponding to all but the $i$-th mode have been made on the matrix $a_{r s}$, the remaining matrix is. $a_{r s}^{(i)}$ from whose rows the $i$-th mode can be found immediately.

\title{
A NORM CRITERION FOR NON-OSCILLATORY DIFFERENTIAL EQUATIONS*
}

BY AUREL WINTNER (The Johns Hopkins University)

Let $f(t), x(t), \lambda(t), \cdots$ denote real-valued, continuous functions on an unspecified half-line, $t_{0} \leqq t<\infty$. If $\lambda(t)$ is positive on this half-line, put

$$
\lambda^{*}=\lambda^{*}(t)=\lambda(t) \int_{t}^{\infty}(d u) / \lambda^{2}(u),
$$

provided that the second factor on the right of (1) is a convergent integral. Under this proviso, a direct substitution of (1) shows that, if $\lambda(t)$ is a solution of the differential equation $D_{f}(\lambda)=0$, where 


$$
\left.D_{f}(\lambda)=D_{f}(\lambda(t))=\lambda^{\prime \prime}(t)+f(t) \lambda(t), \quad{ }^{\prime}=d / d t\right)
$$

then $D_{f}\left(\lambda^{*}\right)=0$, i.e., that $\lambda^{*}(t)$ represents another (linearly independent) solution.

Following A. Kneser ${ }^{1}$, let the differential equation $D_{f}(x)=0$ be called oscillatory or non-oscillatory according as each or none of its solutions $x(t) \not \equiv 0$ has zeros clustering at $t=\infty$. This alternative is complete, since, in view of Sturm's separation theorem, either every or no solution $x(t) \not \equiv 0$ of $D_{f}(x)=0$ has an infinity of zeros on the halfline $t \geqq t_{0}$. The decision of the alternative (for a given coefficient function, $f=f(t)$, of $\left.D_{f}\right)$ is fundamental in certain questions of stability and related applications ${ }^{2}$.

It seems to be of both theoretical and practical interest that the decision can always be based on a criterion similar to the "norm" conditions in the theory of linear functionals and operators (Lebesgue-Toeplitz). It is a criterion the applicability of which does not involve, in principle, the knowledge of a solution $x(t) \not \equiv 0$ of $D_{f}(x)=0$, since it depends on the consideration of arbitrary functions. It can be formulated as follows:

The differential equation $D_{f}(x)=0$ is of non-oscillatory type if and only if there exists some positive function, say $\lambda(t)$, corresponding to which the assignments (1), (2) define two continuous functions the product of which is absolutely integrable, i.e.,

$$
\int^{\infty} \lambda^{*}\left|D_{f}(\lambda)\right| d t<\infty .
$$

As an illustration of how to apply this criterion, choose the arbitrary function $\lambda(t)$ to be $t$. Then (1) and (2) reduce to $\lambda^{*}=1$ and $D_{f}(\lambda)=f(t) t$, respectively, and so (3) will be satisfied if $|f(t)| t$ has a finite integral over the half-line. It follows that the absolute integrability of $f(t) t$ (which, incidentally, is compatible with lim $\sup f(t) t=$ $\infty$ and $\lim \inf f(t)=-\infty$, where $t \rightarrow \infty)$ is sufficient in order that the differential equation $D_{f}(x)=0$ be of non-oscillatory type.

Actually, this particular sufficient condition is contained in an asymptotic result of Bôcher ${ }^{3}$. But this is not a necessary condition. In fact, other sufficient conditions result if the choice $\lambda=t$ is replaced by other choices of the arbitrary function $\lambda(t)$. Such choices can be made relative to the coefficient function, $f$, of $D_{f}$, rather than in a way which, as in $\lambda=t$, is independent of $f$.

Proof of the sufficiency. This part of the italicized criterion can be deduced from the following fact, which is a corollary of a general theorem ${ }^{4}$ : If $p=p(t) \neq 0$ and $q=$ $q(t)$ are continuous functions for large positive $t$, then the condition

$$
\int^{\infty}|q(t)|\left(\int_{t}^{\infty}|p(u)|^{-1} d u\right) d t<\infty
$$

is sufficient in order that some solution $y=y(t)$ of the differential equation

$$
\left(p y^{\prime}\right)^{\prime}+q y=0 \quad\left({ }^{\prime}=d / d t\right)
$$

${ }^{1} \mathrm{~A}$. Kneser, Untersuchungen über die reellen Nullstellen der Integrale linearer Differentialgleichungen, Mathematische Annalen 42, 409-435 (1893), p. 411.

${ }^{2}$ T. v. Kármán and M. A. Biot, Mathematical methods in engineering, New York and London, 1940, Chapter VII and the references on p. 322.

${ }^{3} \mathrm{M}$. Bôcher, On regular singular points of linear differential equations of the second order whose coeffcients are not necessarily analytic, Transactions of the American Mathematical Society 1, 40-52 (1900), pp. 48-52.

${ }^{4}$ A. Wintner, Asymptotic integrations of the adiabatic oscillator in its hyperbolic range, to appear in the Duke Mathematical Journal 15, (1948). 
should tend to a finite limit, as $t \rightarrow \infty$, and that this limit, $y(\infty)$, be distinct from 0 .

It follows that, if $\lambda=\lambda(t)$ is any positive function possessing a continuous second derivative, then the case

$$
p=\lambda^{2}, \quad q=\lambda \lambda^{\prime \prime}+f \lambda^{2}
$$

of (5) must have some solution $y=y(t)$ which does not vanish from a certain $t$ onward, if condition (4) is satisfied by the functions (6). But it is clear from the definitions (1), (2) that the case (6) of (4) is identical with (3). Since $\lambda(t)$ is positive, it follows that (3) implies the non-oscillatory character of that differential equation for $x=x(t)$ which results when $y=x / \lambda$ is substituted into the case (6) of (5).

The result of this substitution is seen to be the differential equation

$$
\left(x^{\prime} \lambda-x \lambda^{\prime}\right)^{\prime}+\left(\lambda^{\prime \prime}+f \lambda\right) x=0 .
$$

Since the latter can be contracted into $\left(x^{\prime \prime}+f x\right) \lambda=0$, where $\lambda>0$, it is equivalent to $x^{\prime \prime}+f x=0$ and so, in view of $(2)$, to $D_{f}(x)=0$. This completes the proof of the sufficiency of (3).

Proof of the necessity. This part of the criterion is of theoretical interest only, and its verification is straightforward. As a matter of fact, $\lambda(t)$ can now be chosen to be a solution $x(t)$ of $D_{f}(x)^{\circ}=0$.

In order to see this, suppose that the differential equation is non-oscillatory. Then there exist a constant $t_{0}$ and a solution $x(t)$ of $D_{f}(x)=0$ such that $x(t)>0$ when $t_{0} \leqq$ $t<\infty$. Let $t^{0}$ be any value exceeding $t_{0}$, restrict $t$ to the half-line $t^{0} \leqq t<\infty$, and put

$$
\lambda(t)=x(t) \int_{t_{0}}^{t}(d u) / x^{2}(u) .
$$

Then $\lambda(t)$ is positive, since $x(t)$ is. Furthermore, it is easily verified from (2) and (7) that $D_{f}(\lambda)=0$, since $D_{f}(x)=0$. Hence, in order to prove that condition (3) is satisfied by the function (7), all that remains to be ascertained is that the function (1) exists in the case (7), i.e., that

$$
\int^{\infty}(d u) / \lambda^{2}(u)<\infty
$$

holds by virtue of (7). But this can be ascertained by an elementary argument used by Hartman ${ }^{5}$.

In fact, it is readily verified from (7) that the Wronskian, $x \lambda^{\prime}-\lambda x^{\prime}$, of $x(t)$ and $\lambda(t)$ is the constant 1 . Hence, the derivative of $x / \lambda$ is identical with $-1 / \lambda^{2}$, and so

$$
x(t) / \lambda(t)=\text { const. }-\int_{t_{\mathrm{o}}}^{t}(d u) / \lambda^{2}(u) .
$$

Since $x(t)>0$ and $\lambda(t)>0$, it follows that

$$
0<\text { const. }-\int_{t_{0}}^{t}(d u) / \lambda^{2}(u) .
$$

This proves (8).

${ }^{5} \mathrm{P}$. Hartman, On differential equations with non-oscillatory eigenfunctions, to appear soon. 